\title{
One-dimensional modelling of the thinning of particulate suspensions near pinch-off
}

\author{
Mariano Rubio-Rubio ${ }^{\mathrm{a}, *}$, Wouter Mathues ${ }^{\mathrm{b}}$, Alejandro Sevilla ${ }^{\mathrm{c}}$, Christian \\ Clasen $^{\mathrm{b}}$ \\ ${ }^{a}$ Área de Mecánica de Fluidos, Departamento de Ingeniería Mecánica y Minera, \\ Universidad de Jaén, Campus de las Lagunillas, 23071 Jaén, Spain \\ ${ }^{b}$ Department of Chemical Engineering, KU Leuven - University of Leuven, Leuven B-3001, \\ Belgium \\ ${ }^{c}$ Departamento de Ingeniería Térmica y de Fluidos, Universidad Carlos III de Madrid, \\ 28911 Leganés, Spain
}

\begin{abstract}
This paper deals with the thinning dynamics of a liquid bridge of dilute particulate suspensions in a Newtonian matrix. We describe the final stage before break-up of a colloidal suspension, which occurs in a liquid thread between two confining beads. The model, based on the one-dimensional mass and momentum conservation equations, shows quantitative agreement with the experimentally observed deceleration and final pinching behaviour of the medium between individually monitored particles in the suspension (Mathues et al., Phys. Fluids 27, 093301, 2015) and thus supports the hypothesis that an accelerated thinning of the Newtonian matrix is caused by an active stretching via independently moving particles.
\end{abstract}

Keywords: Colloidal suspension, liquid bridge, capillary break-up, stretching, $1 \mathrm{D}$ modelling

\footnotetext{
${ }^{*}$ Corresponding author

Email addresses: mrubio@ujaen.es (Mariano Rubio-Rubio), alejandro.sevilla@uc3m.es (Alejandro Sevilla), christian.clasen@kuleuven.be (Christian Clasen)
} 


\section{Introduction}

Fluids used in applications such as inkjet printing, spraying, or dispensing operations in the alimentary or pharmaceutical industries often exhibit a complex rheological behaviour (Chen, 2009). Thus, an operation as simple as the accurate dosing of a liquid can be a problem depending on the fluid properties (Clasen et al. 2012). The aforementioned complexity can arise at two different levels. On the one hand via the development of a microstructure at the molecular level, which can become sufficiently large to modify the macroscopic properties of the fluid. On the other hand via structures present at the mascroscopic level that can have, obviously, extensive influence on the fluid behaviour. The presence of solid particles suspended in a viscous liquid is a clear example of the latter. Suspensions are ubiquitous in nature and industry, with many different examples such as blood, paints and waste slurries. Inks used in daylife printing operations contain solid particles that can modify the normal dripping and jetting response (Basaran et al., 2013) and every novel printing application recently developed or in progress has to deal with the jetting of suspensions (Hoath et al., 2014). However, the basic knowledge about the detailed dynamics of drop and jet formation with these kind of complex fluids is, at least, a step behind their Newtonian counterpart.

After the pioneering work of Nicolas (2002) on gravity-driven suspension jets, the first authors to identify different stages in the pinch-off of a suspension were Furbank and Morris (2004, 2007). They defined an early stage in which the suspension behaves like a continuum medium, characterised by an effective viscosity. While the presence of particles increases this viscosity, experimental measurements suggest that in the second regime the thinning of the ligament no longer follows the behaviour predicted by the effective viscosity, but is accelerated due to effects associated with finite particle size. Precisely this second regime was thoroughly studied by Lidner and coworkers (Bonnoit et al., 2012 Bertrand et al., 2012, Van Deen et al., 2013) with a large set of dripping experiments of polystyrene beads in silicone oil, varying the concentration and 
the particle size. Specifically, they looked at the evolution of the minimum diameter, and compared it with several Newtonian oils of different viscosities, establishing that the presence of particles accelerates the break-up. More recent and thorough experiments due to Mathues et al. (2015) revealed that the neck thinning process decelerates again during the final stages prior to break-up and approaches the behaviour of the matrix fluid close to the pinch-off event. Until recently (Lidner et al., 2015; Zhao et al., 2015), this deceleration stage had not been detected in experimental studies.

The previous experimental works mentioned above can be divided into two different groups, depending on the flow configuration. The first group reproduces the drop formation process by performing dripping experiments, while the second group creates liquid bridges as a model configuration to characterise the break-up into drops. The most prominent example of the use of a liquid bridge as a model system to characterise the thinning and break-up of filaments is the Capillary Breakup Extensional Rheometer, CaBER. A sample of liquid is held between two rods, that are pulled apart to create a slender unstable filament whose temporal evolution allows to extract material properties of the fluid, such as the extensional viscosity of Newtonian liquids (McKinley and Tripathi, 2000), the longest extensional relaxation time in case of visco-elastic fluids (see e.g. Plog et al., 2005, Clasen, 2010, De Dier et al., 2013), or the finite extensibility parameters of elastic components (Szabo et al., 2012).

In the case of thinning and break-up of suspension filaments, the accelerated stage previously described arises initially from variations in local particle density. As the filament becomes thinner, these variations are amplified, leading ultimately to sections of the filament containing no particles. Those sections with an increasingly lower particle density have lower effective viscosities and therefore the thinning dynamics are accelerated. To test this hypothesis, McIlroy and Harlen (2014) made use of a simple one-dimensional model in which the viscosity is determined from the local particle density, found by tracking individual particles within the suspension. In the latter model, the solids only contribute to the dynamics through the local viscosity, so the direct effects of 
hydrodynamic interactions and effects of the individual particles on the free surface are not included. Nonetheless, their model is able to qualitatively reproduce the accelerated thinning found in the experiments, as shown by Mathues et al. (2015), where the self-similar Newtonian scalings are used to support the hypothesis of a solvent-governed break-up. Nevertheless, the final self-accelerated stage is not properly described by the numerical simulations of McIlroy and Harlen (2014) in particular for the important case of small particles and high concentrations, and therefore deserves further analysis. For this thinning stage Mathues et al. (2015) brought up the hypothesis that the second part of the acceleration is caused by an active stretch of the particle-free liquid between two independently moving particles or particle clusters. This was also motivated by the fact that in the final decelerated zone right before break-up both the fitted viscosity from the experimental thinning data and the rescaled profiles coincide again with the bulk liquid properties, but now of the Newtonian medium. However, the number of experimental points gathered during this stage is quite limited and quantitative simulations are so far missing, so additional evidence would be desirable.

The purpose of this work is therefore to model the final thinning stages of the Newtonian liquid trapped between two particles of finite diameter that stretch the liquid bridge with a non-uniform but experimentally exactly prescribed movement. The starting point of the present modelling effort is the work of McIlroy and Harlen (2014), which shows why initially randomly distributed particles lead to local viscosity variations that amplify into regions of accelerated thinning and eventually lead to particle-free filaments. Precisely this late stage, which was suggested to be solely solvent-governed by Mathues et al. (2015) is modelled here numerically, thereby complementing their work. The paper is structured as follows: in Section 2 we describe the flow configuration and the mathematical model employed. The numerical procedure is explained in Section 3 , and the results are presented in Section 4. Finally, the conclusions are summarised in Section 5 


\section{Problem definition}

\subsection{Physical description}

We consider the same configuration studied by Mathues et al. (2015), i.e. a liquid bridge of dilute suspensions of particles dispersed in a Newtonian matrix. In their experiments, capillary break-up experiments were performed with a CaBER-1 extensional rheometer from Thermo Scientific. Sample volumes of the suspensions were loaded between two circular disks, which were later separated to create an unstable liquid bridge. The evolution of the filament was monitored with a high-speed camera, equipped with a lens system that provides resolution enough to identify individual particles in the suspension filament. More details of the experimental procedure can be found in Mathues et al. (2015), and the setup is thoroughly described in Mathues (2015).

Once the suspension sample is stretched, it follows the evolution qualitatively described in Section 1. When the concentration fluctuations lead to a particlefree section in the filament, the interstitial fluid thins until the break-up occurs between two axially separating end particles, which can be clearly identified as in Figure 1. This process resembles a small-scale stretching of a Newtonian filament (Anna et al. 2001), in which the particles play the role of moving endplates. Thanks to the spatial resolution of the experiments, the particles can be tracked over time, providing the stretching velocity profile of the fictitious boundaries associated with the continuous phase thinning.

The suspensions used consisted of non-Brownian spherical particles, dispersed in polydimethylsiloxane oils, whose properties are summarised in Table 1 . As a reference case, a suspension of volume fraction $\phi=10 \%$ of polystyrene spheres with radius $R_{p}=20 \mu \mathrm{m}$, in the following denoted 10PS20, suspended in a PDMS oil of viscosity $\eta=360 \mathrm{mPa}$, shall be considered. Other systems are also studied in the present work, varying the particle radius, the concentration, and the matrix viscosity, as discussed in Subsection 4.3 . 

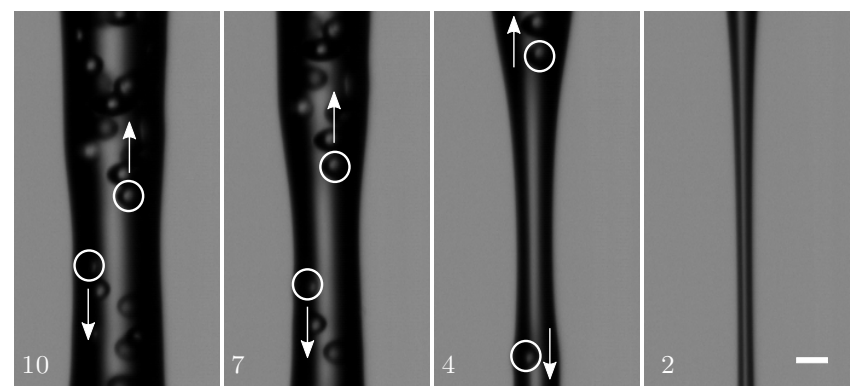

Figure 1: Magnified images of the final stages of the capillary break-up of $10 \%$ PS20 in PDMS2. The number in the bottom left corner indicates the time to pinch-off $\bar{t}_{p}-\bar{t}$ in ms and the scale bar represents $50 \mu \mathrm{m}$. The final thinning takes place between two particles which are highlighted by white circles. Figure adapted from Mathues et al. (2015).

\begin{tabular}{cccc}
\hline & $\eta[\mathrm{mPa} \mathrm{s}]$ & $\rho\left[\mathrm{kg} \mathrm{m}^{-3}\right]$ & $\sigma\left[\mathrm{mN} \mathrm{m}^{-1}\right]$ \\
\hline PDMS 1 & 180 & 1070 & 21.0 \\
PDMS 2 & 360 & 970 & 21.0 \\
\hline
\end{tabular}

Table 1: Properties at $25^{\circ} \mathrm{C}$ of the Sigma-Aldrich silicone oils used as suspension matrices.

\subsection{Mathematical model}

To describe the temporal evolution of the small-scale stretching filament, the fluid trapped between the two end particles is modelled as a Newtonian liquid bridge. Therefore, a liquid of constant density $\rho$, surface tension coefficient $\sigma$, and dynamic viscosity $\eta$ is considered, corresponding to the fluid used as the suspending medium. The liquid is held between two circular plates of radius $R$, which move vertically with velocities $\bar{v}_{0}(\bar{t})$ and $\bar{v}_{L}(\bar{t})$, in presence of the gravitational field $g$. Thus, the distance between the plates $\bar{L}(\bar{t})$ is a function of time. A sketch of the flow configuration can be observed in Figure2.

Considering the liquid bridge axisymmetric, and neglecting the effect of the surrounding atmosphere, the local radius $\bar{h}(\bar{z}, \bar{t})$ and axial velocity $\bar{u}(\bar{z}, \bar{t})$ can be modelled using the one dimensional equations for mass and momentum con- 


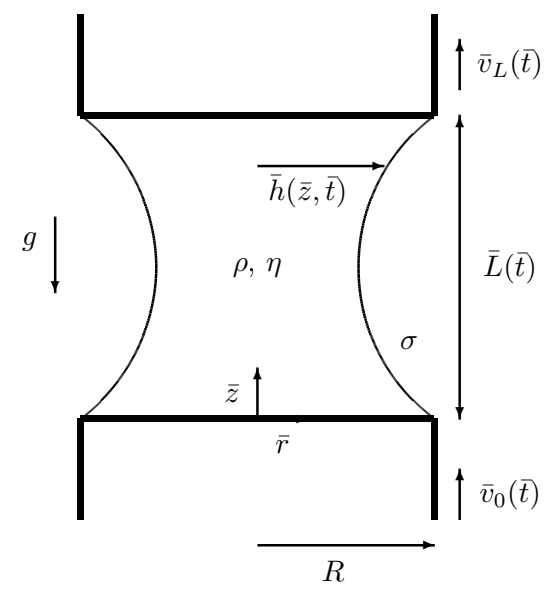

Figure 2: Sketch of the flow configuration and the variables used in the model.

servation derived by Eggers and Dupont (1994),

$$
\begin{aligned}
\frac{\partial h^{2}}{\partial t}+\frac{\partial\left(h^{2} u\right)}{\partial z} & =0, \\
\frac{1}{O h^{2}}\left(\frac{\partial u}{\partial t}+u \frac{\partial u}{\partial z}\right) & =-\frac{\partial \mathcal{C}}{\partial z}+\frac{3}{h^{2}} \frac{\partial}{\partial z}\left(h^{2} \frac{\partial u}{\partial z}\right)-B o\left(1+\frac{a_{0}}{g}\right),
\end{aligned}
$$

where the curvature $\mathcal{C}$ in the pressure gradient term is given by

$$
\mathcal{C}=h^{-1}\left[1+\left(\frac{\partial h}{\partial z}\right)^{2}\right]^{-1 / 2}-\frac{\partial^{2} h}{\partial z^{2}}\left[1+\left(\frac{\partial h}{\partial z}\right)^{2}\right]^{-3 / 2},
$$

retaining its full expression that includes both the radial and the axial terms.

The variables in (1)-(3) have been made dimensionless using the plate radius as characteristic lengthscale, $z_{c}=R$, and the viscous-capillary velocity $u_{c}=\sigma / \eta$ as the velocity scale. Thus, the system (1)-(2) only depends on the Ohnesorge number, $O h=\eta / \sqrt{\rho \sigma R}$, the Bond number $B o=\rho g R^{2} / \sigma$, and the non-dimensional inertia $a_{0} / g$, being $a_{0}=\mathrm{d} \bar{v}_{0} / \mathrm{d} \bar{t}$, which must be taken into account because of the election of the accelerating Eulerian frame moving with the lower end-plate. Notice that throughout this paper, barred symbols denote dimensional variables.

The choice of the one-dimensional model (1)-3 is motivated by its well known accuracy to describe the main features of slender free-surface flows, despite its simplicity. In particular, regarding the momentum equation, it should 
be highlighted that 2 is the simplest one-dimensional approximation that accounts for the main physical mechanisms involved in the thinning process, i.e. inertial, viscous, gravitational and surface tension forces.

The contact line is assumed to be pinned to the plates, $h(0, t)=h(L, t)=1$, and in the moving frame of reference considered, the bottom plate is steady $u(0, t)=0$, while the upper one moves with a velocity $u(L, t)=v=v_{L}(t)-v_{0}(t)$.

As an initial condition for the temporal evolution, the simplest choice would be to consider the bridge as a static cylinder, namely $h(z, 0)=1$ and $u(z, 0)=0$.

\section{Numerical procedure}

To solve the equations (1)-(2) numerically, the method of lines described in Furlani and Hanchak (2011) has been implemented. Accordingly, the spatial derivatives are discretised using a second-order finite difference scheme, and the time derivatives can then be solved as a set of ordinary differential equations for the discretised radii and velocities.

Although the spatial domain evolves over time due to the plate movement, a fixed computational domain $0 \leq \xi \leq 1$ is used to ease the implementation of the spatial derivatives and also to avoid the need of an adaptative method. To that end, the instantaneous domain length is used as a rescaling parameter of the form $\xi=z / L(t)$. This mapping introduces in the equations $\sqrt{1}-(3)$ the metrics given by the chain rule which, for the $i$-th spatial derivative, reads $\partial_{z}^{i}=1 / L^{i} \partial_{\xi}^{i}$. The time derivatives are also modified, yielding $\partial_{t}=\partial_{\tau}-(v / L) \xi \partial_{\xi}$, being $\tau=t$ the computational time, which is not rescaled.

To increase the numerical stability of the method, two staggered grids are jused for the computation of the radii and the velocities, as suggested by Furlani and Hanchak (2011). Thus, the local radius $h$ is computed at $n$ equidistant points in the computational domain, while $n-1$ velocity nodes are uniformly placed interlaced midway between those defined for $h$ as shown in Figure 3

The temporal evolution is solved using Matlab ${ }^{\circledR}$ 's built-in ODE15s function, which is a variable order, multistep, implicit solver, capable of handling stiff 


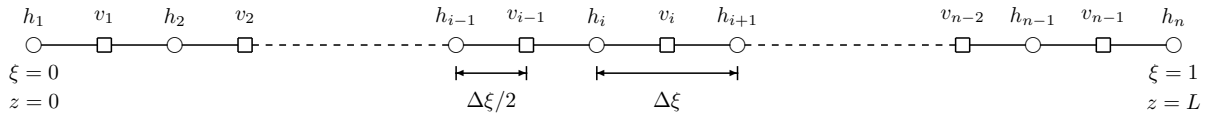

Figure 3: Staggered computational grid.

170

problems.

The code was tested using two particular cases, i.e. the static shape of a portion of fluid held between two plates, and the case of a liquid bridge stretched at a constant velocity. The simulation results coincide with the well known profile of the static bridge for the former while in the latter case, we were able to reproduce the results of Zhang et al. (1996), obtaining excellent agreement. In addition, grid tests were performed to ensure independence of the numerical results on the discretisation. All the simulations reported in Section 4 are computed using $n=200$, and the relative error in volume conservation is less than $0.6 \%$ for the reference case mentioned above. The number of timesteps is $n_{t} \sim 500$ to provide smooth evolutions of the shapes and velocity fields. With this set of parameters, the time needed to perform a simulation is less than 3 minutes in a standard desktop computer.

\section{Results}

\subsection{Constant plate radius}

To check the validity of the model proposed in the present work, we compare the numerical results with the experiments reported in Mathues et al. (2015). To that end, one representative run of the 10PS20 suspension in PDMS2 is chosen as the reference case. Here, the values of the governing parameters are $O h=9.6$ and $B o=2.1 \times 10^{-3}$. For this case, the velocity $v$, and the dimensionless inertia $a_{0} / g$ are represented as a function of time to pinch-off $t_{p}-t$ in Figure 4(a). With these inputs, the comparison between the numerically computed and the experimental evolution of the minimum radius of the stretching filament is plotted in Figure 4(b). From the results shown in Figure 4(b) it can be deduced that although the trend of the simulation is the same as in the 

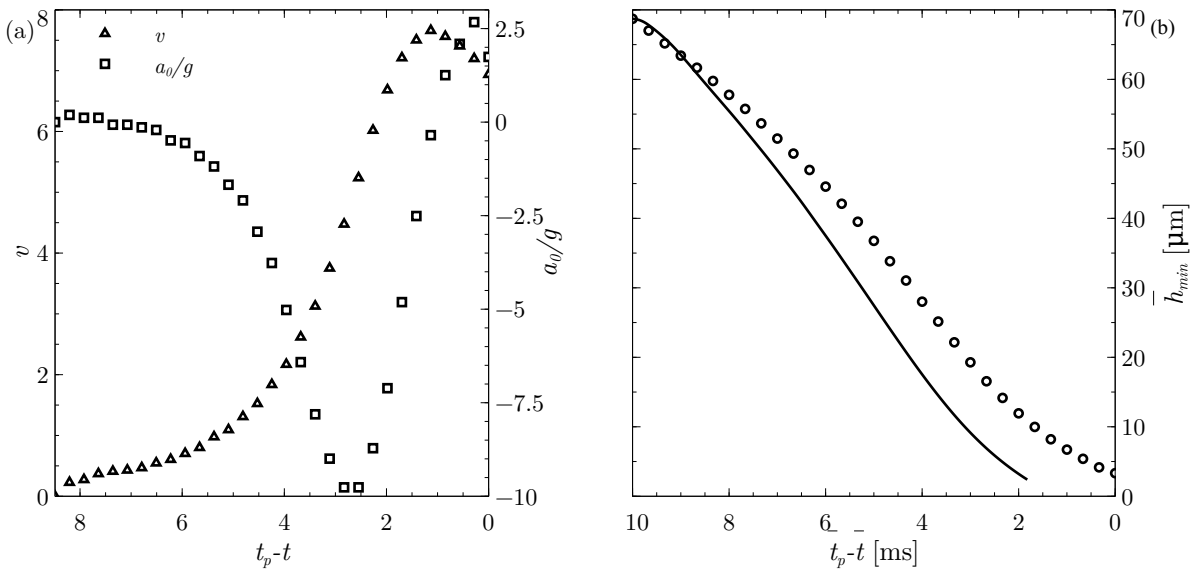

Figure 4: (a) Experimental velocity profile evolution, $\Delta$ (left scale) and dimensionless inertia $a_{0} / g, \square$ (right axis), as functions of time to pinch-off $t_{p}-t$ used as input parameters for the model to calculate the case of $10 \%$ PS20 particles in PDMS oil with $\eta=360 \mathrm{mPa}$ s. (b) Comparison of the numerical (solid line) and experimental (symbols) minimum radius for the same case.

experiment (obtaining a good qualitative agreement), there are differences in magnitude, leading to a faster pinching of the numerical solution.

The deviations between the simulation and the experiment can be traced back to the assumptions made for the modelling. In particular, when the local filament radii at the end particle positions are carefully observed in Figure 1 it is clear that both decrease with time. Hence, the initial assumption of constant plate radii in the model is invalid. An assumed pinned contact line with a radius larger in the simulation than in the experiment implies that, for the same liquid volume, the hourglass-like shape of the bridge is more pronounced in the former case. In turn, the radial curvature of the interface is larger in the model than in the real case. It is well known (Plateau, 1873, Rayleigh, 1878) that an incremented radial curvature in a liquid column results in a destabilising effect: the rise of the capillary pressure in the neck radius drains fluid axially from the local minimum of the filament, leading to the pinch-off. The latter argument explains why in Figure 4 (b) the simulated bridge thins faster than the experimental one, resulting in an advanced break-up of the modelled filament. 


\subsection{Linearly decreasing plate radius}

To confirm that the origin of the quantitative disagreement between the simulation and the experiment is caused by the hypothesis of a constant radius of the virtual end plates, the influence of a decreasing plate radius on the thinning dynamics has also been studied. To that end, the boundary conditions were modified, first by imposing the simplest evolution of the boundary, a linear decrease with time. In dimensional variables, this corresponds to $R(\bar{t})=R_{0}$ $\left(R_{f}-R_{0}\right) \bar{t} / \bar{t}_{p}$, with $R_{f}$ being the plate radius when the filament pinches off at time $\bar{t}_{p}$. In Figure 5(a), the evolution of the minimal radius over time is presented for such a linear decrease of the virtual plate with $R_{f}=30 \mu \mathrm{m}$. As can be observed, the decreasing size of the plates improves the agreement between the simulation and the experiment, demonstrating that the hypothesis of fixed boundary condition is too restrictive. Nevertheless, the use of such a simple law entails several drawbacks. At the early times, when the stretching velocity is small, the evolution of the minimum radius coincides with the instantaneous plate size, instead of the neck of the filament. Thus, the simulated shapes during these initial stages are more similar to a barrel than to an hourglass. The inset of Figure 5(a) shows a detail of the initial stage, in which a change in the slope of the computed evolution is noticeable, corresponding to the unphysical result mentioned above. In addition, $R_{f}$ is a free parameter of the model, whose value can be selected to improve the agreement between the numerical and experimental results. In summary, the minimum radius of the liquid bridge is better reproduced when considering that the end plates shrink with time, but the simplicity of a linearly decreasing law implies that the shape of the whole filament is not properly captured in all the spatial domain, specially near the ends. An example can be found in Figure $5(\mathrm{~b})$.

\subsection{Experimentally determined plate radius}

To improve the agreement with the experiments, as well as to eliminate any free parameter, the model can be revised to simulate more realistic conditions. Thus, in the present subsection, instead of supposing a linear decrease of the 


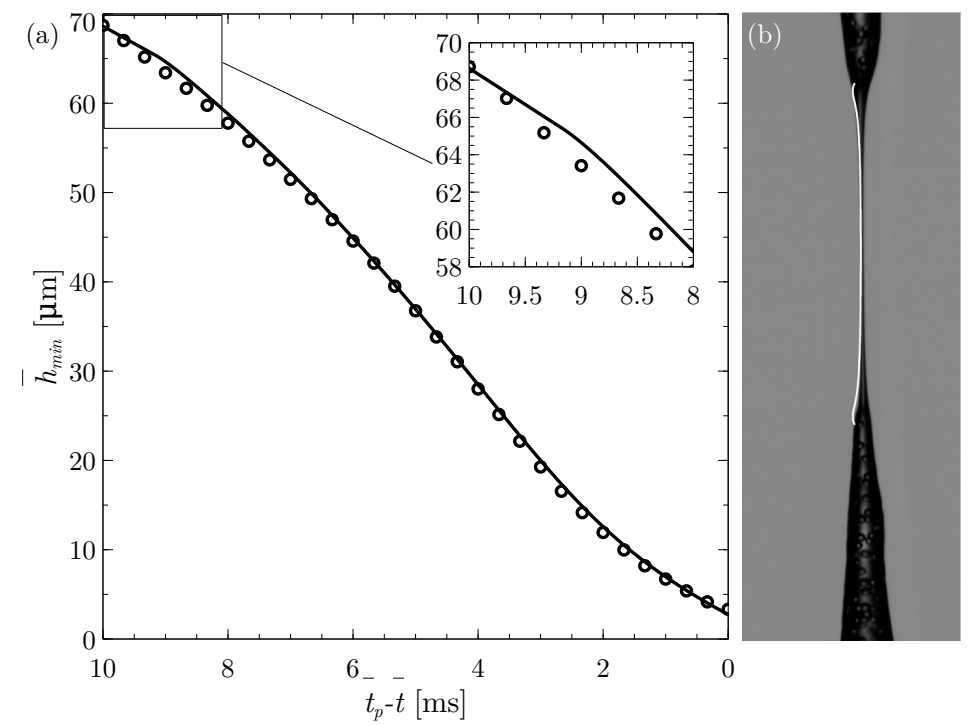

Figure 5: (a) Comparison between the time evolution of the experimental (symbols) and the numerical (solid line) minimum diameter for the reference case using a linearly decreasing size of the plates as a boundary condition with $R_{f}=30 \mu \mathrm{m}$. The inset shows a zoom of the initial instants. (b) Comparison between the computed and the experimental shape at $\bar{t}_{p}-\bar{t}=1.0$ ms. 


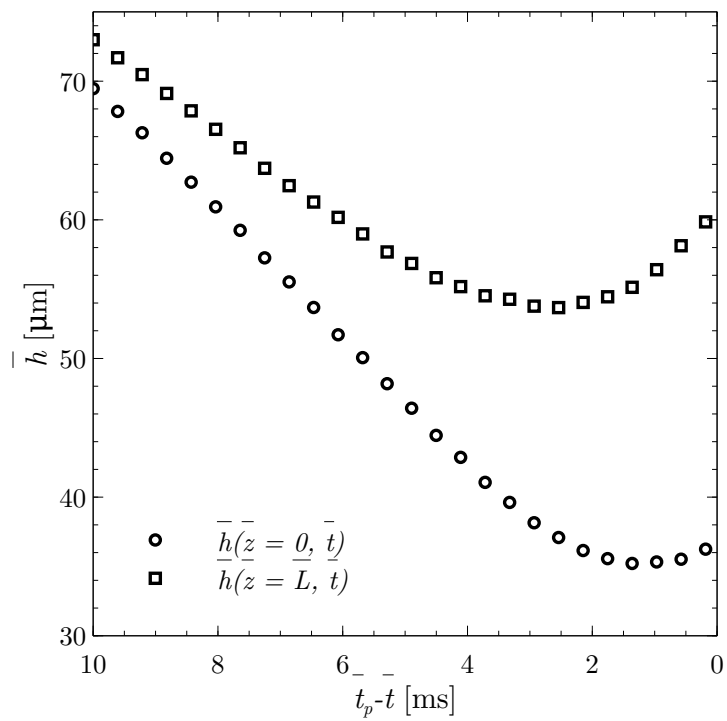

Figure 6: Temporal evolution of the experimental radii at the positions of the end particles in the reference case. Squares represent the top plate radius, and circles the bottom one.

plate radii, the real evolution of the radius at both ends, $\bar{h}(0, \bar{t})=\bar{h}_{\text {exp }}(0, \bar{t})$ and $\bar{h}(\bar{L}, \bar{t})=\bar{h}_{\text {exp }}(\bar{L}, \bar{t})$ is imposed. As can be observed in Figure 6, where these temporal evolutions are plotted for the reference case, the boundary conditions are far from being constant. Moreover, it can be appreciated that they are not equal for both plates, although their trends are similar.

Indeed, Figure 6 shows that $\bar{h}(0,0) \neq \bar{h}(\bar{L}, 0)$, so the initial condition of a cylindrical shape used until this point is no longer valid. Therefore, the real shape of the filament extracted from the experiment has also been used as initial condition for the interface profile $\bar{h}(\bar{z}, 0)=\bar{h}_{\text {exp }}(\bar{z}, 0)$. The computed evolution of the neck radius with these new boundary and initial conditions is shown in Figure 7, where an excellent agreement with the experimental result for the reference case can be noticed.

Moreover, this agreement remains very good when modifying the properties of the suspension. Indeed, in Figure 8, the comparison between numerical simulations and experiments is extended, presenting different cases when varying one property at a time while keeping the rest constant. Thus, the results 


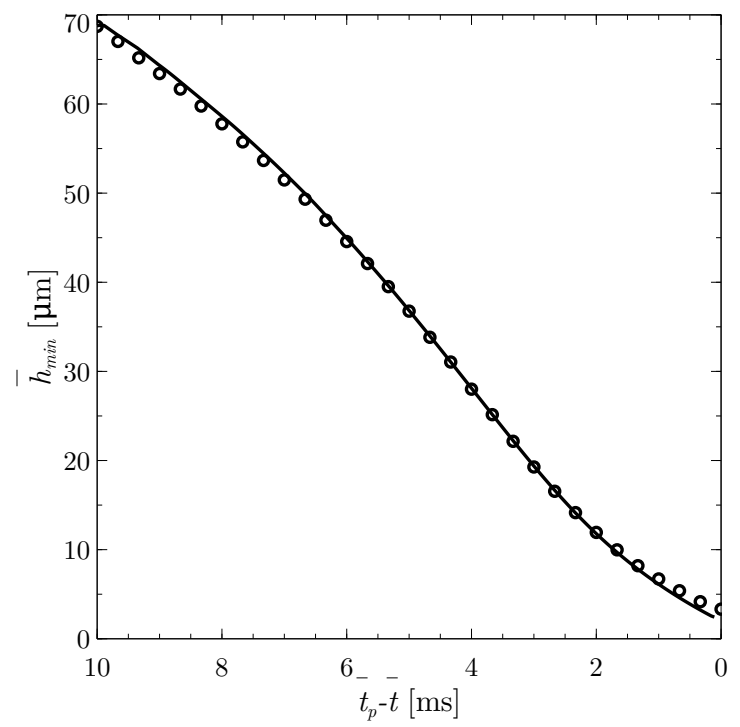

Figure 7: Temporal evolution of the minimum diameter for the case of $10 \%$ PS20 particles in PDMS oil $\eta=360 \mathrm{mPa}$ s, using the experimental profile as initial condition, and the experimental evolutions of the end-plate radii as boundary conditions.

are presented for two different particle concentrations, particle sizes and matrix viscosities, as summarised in Table 2 .

With the change in the boundary condition, the model is not only able to predict the minimum radius between the two end particles, but also the shape of the whole filament. This fact can be observed in Figure 9 and Movie 1 of the supplementary material, where the computed profiles are plotted over the experimental images, for the reference case of $10 \%$ PS particles of radius

\begin{tabular}{cccc}
\hline Variation & $\phi$ & $R_{p}[\mu \mathrm{m}]$ & Matrix \\
\hline Reference & $10 \%$ & 20 & PDMS 2 \\
Concentration & $25 \%$ & 20 & PDMS 2 \\
Particle size & $10 \%$ & 10 & PDMS 2 \\
Matrix & $10 \%$ & 20 & PDMS 1 \\
\hline
\end{tabular}

Table 2: Variations from the reference suspension to extend the comparisons. 


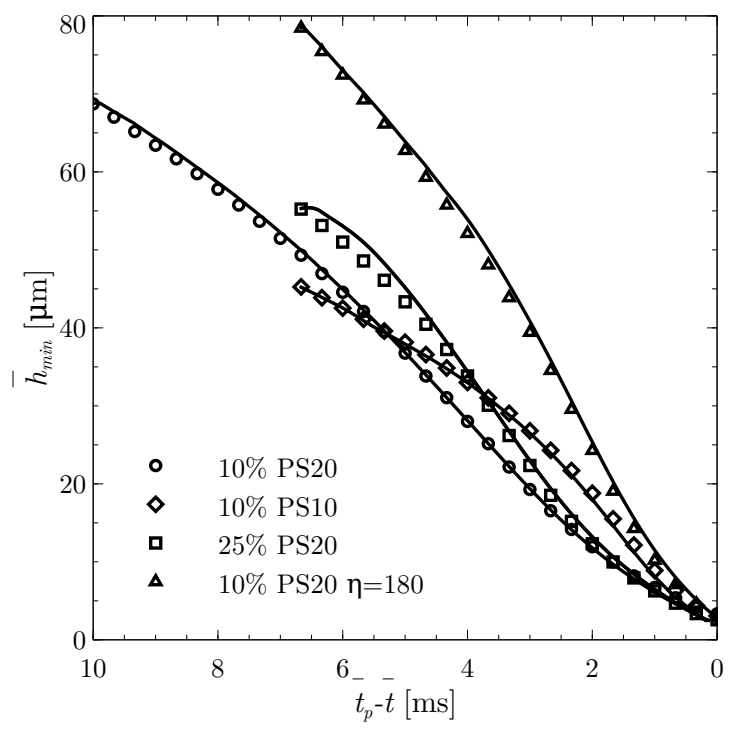

Figure 8: Comparison between the computed (lines) and experimental (symbols) evolution of the minimum diameter for different suspension properties. With $\circ$, the reference case of 10PS20 in PDMS $1 ; 10 \%$ particles of radius $R_{p}=10 \mu \mathrm{m}$ in PDMS oil $\eta=360 \mathrm{mPa} \mathrm{s}, \diamond ; 25 \%$ PS particles of radius $R_{p}=20 \mu \mathrm{m}$ in the same oil, $\square$; and $10 \%$ PS particles of radius $R_{p}=20$ $\mu \mathrm{m}$ in PDMS 2 oil, with $\eta=180 \mathrm{mPa} \mathrm{s}, \Delta$. 


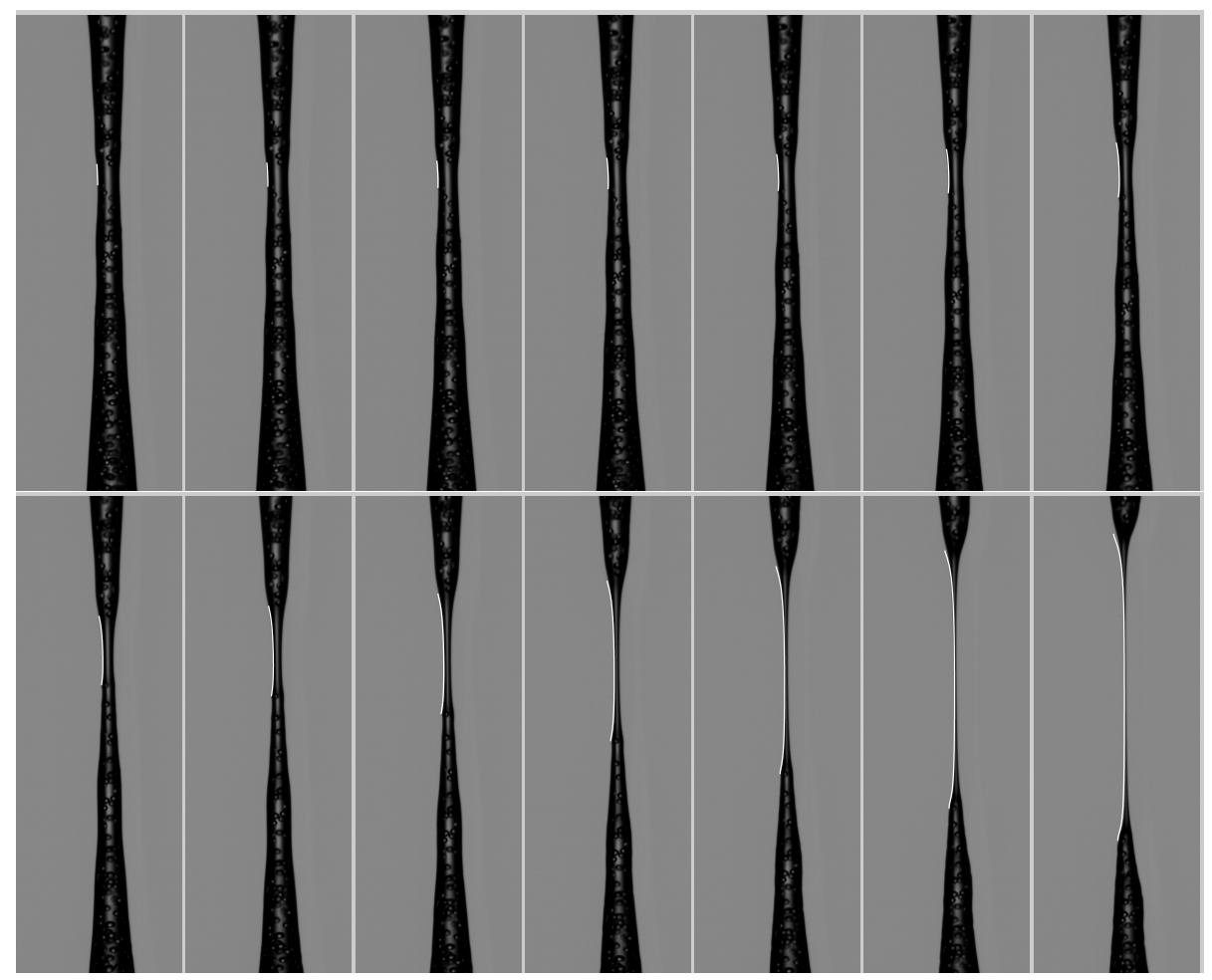

Figure 9: Experimental and numerical shape evolution comparisons for the case of $10 \%$ PS20 particles in PDMS oil $\eta=360 \mathrm{mPa}$. The time between two consecutive frames is $0.666 \mathrm{~ms}$.

$R_{p}=20 \mu \mathrm{m}$ in PDMS oil of viscosity $\eta=360 \mathrm{mPa}$ s. In particular, it can be deduced from the agreement between the simulation and the experiment that the thinning and pinch-off of the suspension is indeed governed solely by the thinning dynamics of the interstitial Newtonian fluid, as was so far only speculated by Mathues et al. (2015), and that the proposed one-dimensional model in this paper is appropriate to describe the process.

Figure 10 shows the comparisons between the simulations and experiments when varying the suspension properties. The dependence of the thinning velocities on the suspension properties were discussed by Mathues et al. (2015). The top row of Figure 10, and the Movie 2 of the supplementary material, show the case of $10 \%$ concentration of particles with radius $R_{p}=10 \mu \mathrm{m}$ in PDMS 2, the smaller size of the particles being clearly noticeable from the images. In the 
middle row, and Movie 3 of the supplementary material, the concentration is different than that of the reference case, namely 25\% PS20 particles in silicone oil of viscosity $\eta=360 \mathrm{mPa}$ s. Finally, the bottom row of Figure 10 , and Movie 4 of the supplementary material, show the case of $10 \%$ PS20 particles in a silicone oil matrix with a different viscosity of $\eta=180 \mathrm{mPa}$ s. In all cases, the simulations correctly predict the shape of the filament during the whole process, provided that the experimental stretching velocities and the radii at both ends are used as boundary conditions.

It should be noticed that, since the model is developed to describe the thinning behaviour of the suspension by considering the particle-free zone, the profiles in figures 9 and 10 are calculated precisely within the two end particles acting as virtual plates. Because of that, specially at early times, the computed profiles represented in white on top of the experimental images are smaller than the whole liquid bridge.

\section{Conclusions}

In this work a simple model to describe the final thinning stage of particulate suspensions has been developed. This final behaviour had not been correctly captured in previous studies, which only provided good descriptions of the thinning dynamics at earlier stages of the process. Considering the interstitial fluid between two end particles as a small-scale Newtonian liquid bridge, the onedimensional mass and momentum equations introduced by Eggers and Dupont (1994) are capable of describing the shape evolution of the stretching filament, provided that the velocities and the radii at both ends extracted from the experiments are used as boundary conditions. The results are in excellent agreement with the experiments for different cases, including variations of the particle size, the concentration and the matrix viscosity. Moreover, the calculations involve an extremely low computational cost, running in a standard personal computer in a few minutes. The results obtained herein clearly demonstrate that the end pinching dynamics of a dilute particulate suspension, which rheologically consti- 


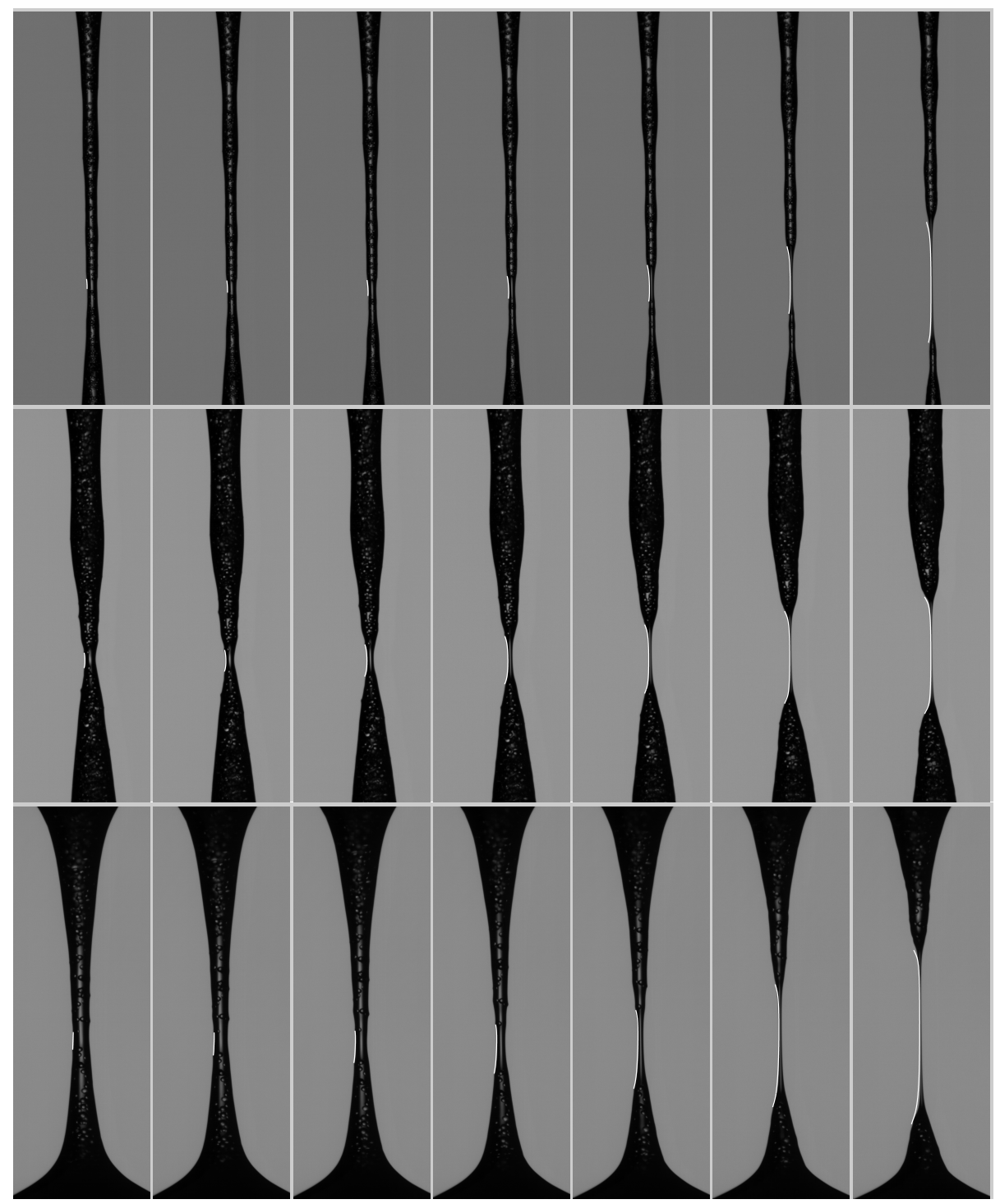

Figure 10: Comparison between the experimental and numerical shape evolution for the cases: $10 \%$ PS10 particles in PDMS oil $\eta=360 \mathrm{mPa}$ s (top row); $25 \%$ PS20 particles in PDMS oil $\eta=360 \mathrm{mPa}$ s (middle row); and $10 \%$ PS20 particles in PDMS oil $\eta=180 \mathrm{mPa}$ s (bottom row). The time between two consecutive frames is $0.666 \mathrm{~ms}$. 
tutes a non-Newtonian system, corresponds solely to break-up of a Newtonian fluid. The simulations confirm that the second stage of the experimentally observed accelerated thinning is indeed caused by the additional stretching of the otherwise simply Newtonian fluid between the two independently moving particles or particle clusters, as hypothesised by Mathues et al. (2015). The thinning and rupture differ therefore qualitatively from the evolution of a destabilised Newtonian liquid bridge between two fixed plates, since the non-uniform movement of the end particles adds an active stretching to the capillary pressure driven thinning.

With the demonstrated convenience of the proposed model to describe the dynamics, a natural extension of this work would be to derive a predictive model. Thus, if both the velocities of the tracked end particles, and the time evolution of the filament radii at these positions could be predicted as functions of the properties of the suspension, the simulations performed in this study would turn independent of the experiments. This modelling effort would have to deal with the non-Newtonian behaviour of the colloidal suspension, which is left for future studies.

\section{Acknowledgements}

MRR and AS thank the financial support of the Spanish MINECO [projects no. DPI2014-59292-C03-01-P and no. DPI2015-71901-REDT]. These research projects have been partly financed through European funds. WM and CC would like to thank support from the ERC starting grant [no. 203043 NANOFIB] and the Research Foundation Flanders [FWO project G077916N].

\section{Appendix}

Model inputs

The boundary conditions, i.e. the size of the end plates and the relative velocity, plus the inertial acceleration of the moving frame of reference are the inputs needed to perform the simulations. In the case of the variations from the 
reference suspension devoted to extend the comparisons to different systems, those inputs are shown in Figure 11. 10\% PS10 particles in PDMS oil with $\eta=360 \mathrm{mPa}$ s in the top row, 25\% PS20 particles in $\eta=360$ oil $\mathrm{mPa} \mathrm{s}$ in Figure 11.(c) and (d), and 10\% PS20 particles in oil with $\eta=180 \mathrm{mPa}$ s in the bottom row.

\section{References}

Anna, S.L., McKinley, G.H., Nguyen, D.A., Sridhar, T., Muller, S.J., Huang, J., James, D.F., 2001. An interlaboratory comparison of measurements from filament-stretching rheometers using common test fluids. J. Rheol. 45, 83-114.

Basaran, O.A., Gao, H.J., Bhat, P.P., 2013. Nonstandard inkjets. Annu. Rev. Fluid Mech. 45, 85-113.

Bertrand, T., Bonnoit, C., Clement, E., Lindner, A., 2012. Dynamics of drop formation in granular suspensions: The role of volume fraction. Granular Matter 14, 169-174.

Bonnoit, C., Bertrand, T., Clément, E., Lindner, A., 2012. Accelerated drop detachment in granular suspensions. Phys. Fluids 24, 043304.

Chen, X.B., 2009. Modeling and control of fluid dispensing process: A state of the art review. Int. J. Adv. Manuf. Technol. 43, 276-286.

Clasen, C., 2010. Capillary breakup extensional rheometry of semi-dilute polymer solutions. Korea-Aus. Rheol. J. 22, 331-338.

Clasen, C., Phillips, P.M., Palangetic, L., Vermant, J., 2012. Dispensing of rheologically complex fluids: The map of misery. AIChE J. 58, 3242-3255.

De Dier, R., Mathues, W., Clasen, C., 2013. Extensional flow and relaxation of semi-dilute solutions of schizophyllan. Macromol. Mater. Eng. 298, 944-953.

Eggers, J., Dupont, T.F., 1994. Drop formation in a one-dimensional approximation of the navier-stokes equation. J. Fluid Mech. 262, 205-222. 

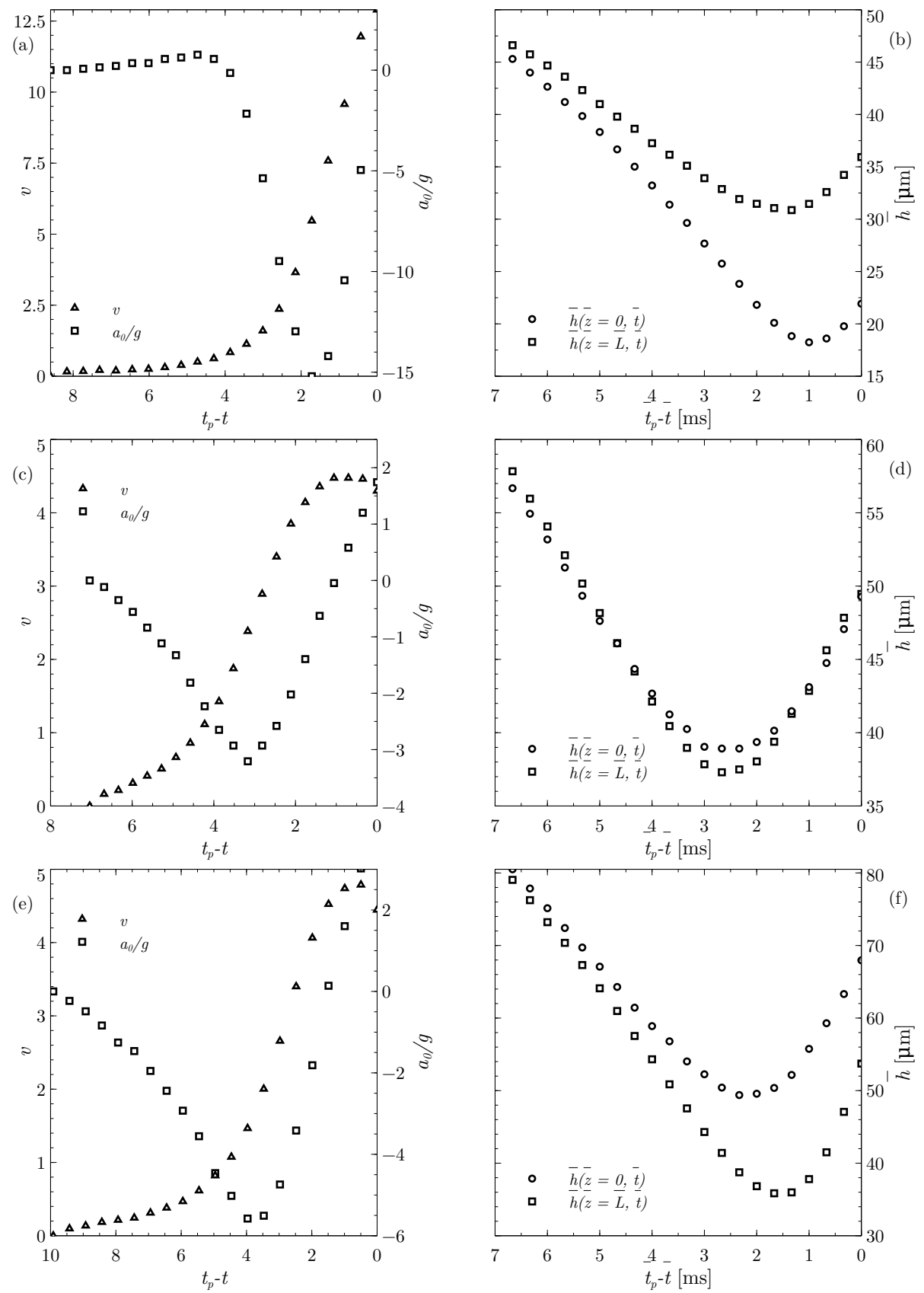

Figure 11: (a)(c)(e) Experimental velocity profile evolution, $\Delta$ (left scale) and dimensionless inertia $a_{0} / g, \square$ (right axis), as functions of time to pinch-off $t_{p}-t$. (b)(d)(f) Temporal evolution of the experimental radii at the positions of the end particles for the same cases. Squares represent the top plate radius, and circles the bottom one. Top row, (a), (b) $10 \%$ PS10 particles in PDMS1 oil; middle row, (c), (d) 25\% PS20 particles in PDMS1 oil; and bottom row, (e), (f) $10 \%$ PS20 particles in PDMS2 oil. 
Furbank, R.J., Morris, J.F., 2004. An experimental study of particle effects on drop formation. Phys. Fluids 16, 1777-1790.

Furbank, R.J., Morris, J.F., 2007. Pendant drop thread dynamics of particleladen liquids. Int. J. Multiphase Flow 33, 448-468.

Furlani, E.P., Hanchak, M.S., 2011. Nonlinear analysis of the deformation and breakup of viscous microjets using the method of lines. Int. J. Numer. Meth. Fluids 65, 563-577.

Hoath, S.D., Hsiao, W.K., Hutchings, I.M., Tuladhar, T.R., 2014. Jetted mixtures of particle suspensions and resins. Phys. Fluids 26, 101701.

Lidner, A., Fiscina, J.E., Wagner, C., 2015. Single particles accelerate final stages of capillary break-up. Europhys. Lett. 110, 64002.

Mathues, W., 2015. Filament stabilisation in free-surface flows of complex fluids. Ph.D. thesis. KU Leuven.

Mathues, W., McIlroy, C., Harlen, O.G., Clasen, C., 2015. Capillary breakup of suspensions near pinch-off. Phys. Fluids 27, 093301.

McIlroy, C., Harlen, O.G., 2014. Modelling capillary breakup of particulate suspensions. Phys. Fluids 26, 033101.

McKinley, G.H., Tripathi, A., 2000. How to extract the Newtonian visosity from Capillary Breakup Measurements in a filament rheometer. J. Rheol. 44, 653-671.

Nicolas, M., 2002. Experimental study of gravity-driven dense suspension jets. Phys. Fluids 14, 3570-3576.

Plateau, J., 1873. Statique Expérimentale et Théorique des Liquides. GauthierVillars et $\mathrm{C}^{i e}$, Paris.

Plog, J.P., Kulicke, W., Clasen, C., 2005. Influence of the molar mass distribution on the elongational behaviour of polymer solutions in capillary breakup. Appl. Rheol. 15, 28-37. 
Rayleigh, W.S., 1878. On the instability of jets. Proc. London Math. Soc. 10, $4-13$.

Szabo, P., McKinley, G.H., Clasen, C., 2012. Constant force extensional rheometry of polymer solutions. J. Non-Newtonian Fluid Mech. 169, 26-41.

Van Deen, M.S., Bertrand, T., Vu, N., Quéré, D., Clement, E., Lidner, A., 2013. Particles accelerate the detachment of viscous liquids. Rheol. Acta 52, 403-412.

Zhang, X., Padgett, R.S., Basaran, O.A., 1996. Nonlinear deformation and breakup of stretching liquid bridges. J. Fluid Mech. 329, 207-245.

Zhao, H., Liu, H.F., Xu, J.L., Li, W.F., Lin, K.F., 2015. Inhomogeneity in breakup of suspensions. Phys. Fluids 27, 063303. 